\title{
On the locus of the speed/accuracy tradeoff*
}

\author{
GEORGE E. BRIGGS and DAVID SHINAR \\ Ohio State University, Columbus, Ohio 43210
}

A statistically significant interaction between visual noise level and accuracy level was obtained for reaction time data in the Sternberg choice reaction task. This suggests that the speed/accuracy tradeoff is localized in the initial stimulus encoding stage of processing, specifically in a stimulus sampling operation. A slower stimulus sampling rate was found under visual noise than was found with a noise-free display.

Swanson \& Briggs (1969) demonstrated that reaction time (RT) in the Sternberg (1966) choice reaction task could be decomposed into three components:

$$
R T=c+d\left(H_{t}\right)+b\left(H_{c}\right)
$$

where $H_{t}$ is information transmitted and $\mathrm{H}_{c}$ is central processing uncertainty. The fitted constants were:

$$
\mathrm{RT}=.243+.157\left(\mathrm{H}_{\mathrm{t}}\right)+.108\left(\mathrm{H}_{\mathrm{c}}\right)
$$

They interpreted $b\left(\mathrm{H}_{c}\right)$ as the time per bit to classify the test stimuli as to positive or negative set status, a Stage 2 or central processing function in the Smith (1968) sequential-stage paradigm of human information processing. They interpreted $d\left(H_{t}\right)$ as the time per bit required to sample stimulus information from a short-term sensory store, a Stage 1 or initial stimulus encoding function in the Smith model. Finally, c would seem to be the average time to complete all other information processing functions, particularly the Stage 3 function of response decoding.

It must be admitted that the interpretation of $d\left(\mathrm{H}_{t}\right)$ was not based on hard evidence. It was not possible for Swanson \& Briggs (1969) to show, other than by logic, that this index of the speed/accuracy tradeoff was localized in Stage 1 rather than in Stage 3 of the Smith paradigm. A subsequent study by Briggs \& Swanson (1970) did offer some evidence that the earlier interpretation was valid: they did not find an interaction between the accuracy level, which gives rise to $d\left(H_{t}\right)$, and response load. The latter presumably influences the Stage 3 response decoding function, and from the logic of the Sternberg

*This study was sponsored in part by the National Science Foundation through Grant GN-534.1 from the Office of Science Information Service to the Computer and Information Science Research Center, Ohio State University. Send reprint requests to: George E. Briggs, Human Performance Center, 404B West 17th Avenue, Columbus, Ohio 43210 .
(1969) additive-fact or method, independent variables which influence the same process will give rise to a statistically significant interaction. Therefore, this would suggest that the speed/accuracy variable does not influence Stage 3, thereby implicating Stage 1 as the locus of the effect, but this evidence is somewhat indirect.

In a recent address, Sternberg (1971) suggested the need for a more direct test of the locus of the speed/accuracy tradeoff. The present paper describes the results of such a test.

Research by both Sternoerg (1967) and Bracey (1969) has demonstrated that the primary effect of visual noise is on the initial stimulus encoding stage of processing. Therefore, a factorial combination of two levels of visual noise and two levels of accuracy should give rise to an interaction of these two variables if the speed/accuracy tradeoff is localized in Stage 1.

\section{Design and Procedure}

Forty eight Ss (20 males and 28 females) were assigned, in order of appearance, to one of four groups. Groups were defined by the factorial combination of speed ( $15 \%$ errors) vs accuracy (5\% errors) and two levels of visual noise (a noise-free display vs a checkerboard pattern superimposed on the display, which partially obscured the test stimuli). Each $S$ received two daily sessions of practice at the Sternberg (1966) choice reaction task to stabilize performance at either the $5 \%$ or the $15 \%$ level, as appropriate, and then each $S$ contributed data for analysis in two final daily sessions. The Ss received $\$ 1.25 /$ session (approximately $30 \mathrm{~min}$ in duration), plus a bonus of 20 cents for every 32-trial block in which their performances approximated the accuracy criterion. The $S$ was never told that he was being rewarded for response accuracy; instead, E simply encouraged $S$ to speed up or slow down (depending upon his error rate), and, as far as S knew, the reward was given when his average reaction time was "fast enough" and the errors were not "too many."
A varied set procedure defined the Sternberg task: prior to each block of 32 trials, $E$ read $S$ a memory (M) set of four letters from the ensemble $A, B$, $\mathrm{D}, \mathrm{G}, \mathrm{H}, \mathrm{I}, \mathrm{J}, \mathrm{K}, \mathrm{Q}$, and $\mathrm{Y}$. Whenever a letter was not a member of the positive (memorized) set, it served as a negative set stimulus. As usual, S responded either "match" (of test to one of the $M=4$ positive set stimuli) or "no match" for each of the 32 test stimuli in the ensuing block of trials. In randomized order, half of the test stimuli were from the positive set and half were from the negative set within each block. The membership of the positive set changed from one block to the next, with counterbalancing both between and within $S$ as to letters assigned to the positive sets. There were three blocks of trials per day. An a uditory warning signal occurred. $500 \mathrm{msec}$ prior to a test stimulus, and then the stimulus itself was displayed for $2 \mathrm{sec}$, during which time $S$ responded by pressing one of two buttons ("match" or "no match"). A 3.0-sec intertrial interval ensued prior to the next warning signal.

When utilizing the Sternberg (1966) task, one usually uses two or more sizes of positive set. Only $M=4$ was used here, as it is difficult (and perhaps inappropriate) to achieve comparable accuracy levels across memory load. It was felt that $M=4$, rather than either a larger or a smaller sized positive set, would provide a reasonably difficult task for $S$, especially under visual noise.

\section{APPARATUS}

The $S$ sat approximately $55 \mathrm{~cm}$ before the visual display and rested his right index and middle fingers on the two response buttons. Test stimuli were back-projected on the display from a Kodak Carousel RA950 random access projector. The single letter appeared black on a light field for the noise-free display condition, and, for the visual noise condition, it also appeared as black on white, but it was viewed through a checkerboard pattern mounted directly on the display. The visual noise pattern consisted of a No. 551 Zip-a-Tone sheet manufactured by Para-Tone, Inc. There are approximately three black squares and three white squares per centimeter on this noise pattern. The test stimuli were all $9 \mathrm{~mm}$ tall and between 6 and $7 \mathrm{~mm}$ wide at the display surface, except the letter I which was $1 \mathrm{~mm}$ wide. The segments of each letter were $1 \mathrm{~mm}$ thick also. The letters had been drawn from the Leroy Standard Template and were in uppercase.

Onset of the test stimulus activated an electronic timer accurate to $1 \mathrm{msec}$. The S's depression of either response button stopped the timer, and it 


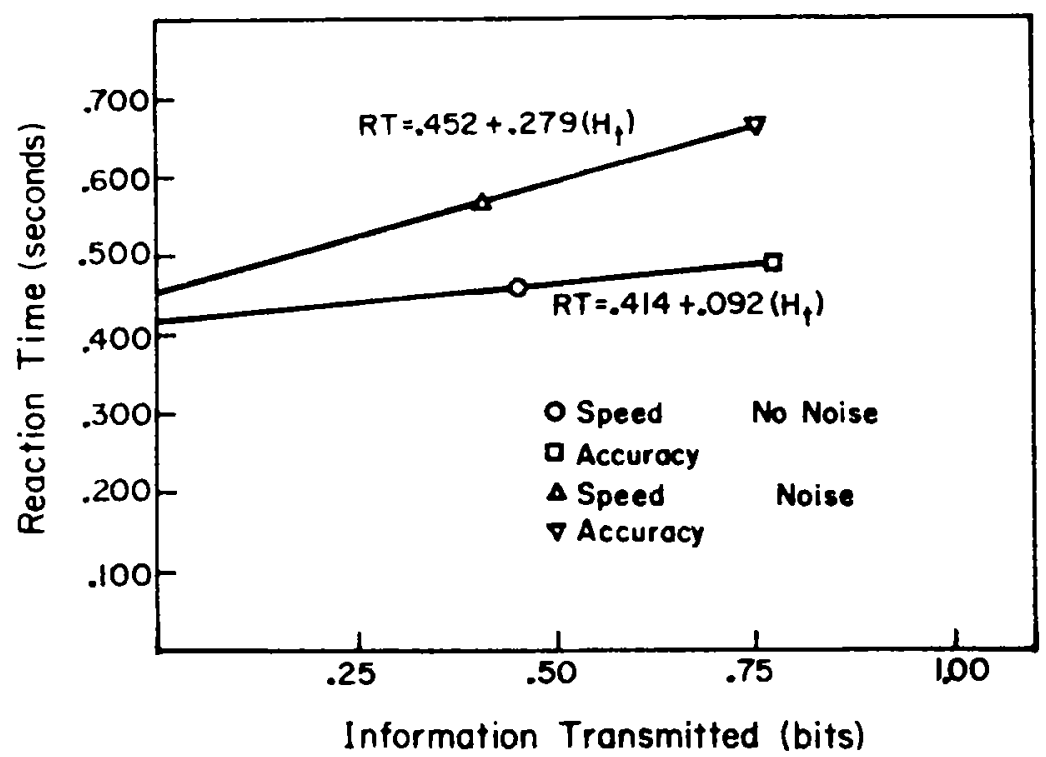

Fig. 1. Speed vs accuracy for the two visual noise levels.

resulted in a printout of the reaction time and the button pressed. The $S$ wore headphones over which $E$ could read the positive set at the beginning of each block and provide the feedback on the bonus at the end of each block. The $S$ heard $80 \mathrm{~dB}$ of white noise to mask extraneous sounds in the laboratory when not in communication with E. A 500-msec interruption of the auditory noise served as the warning signal. Assignment of "match" and "no match" status to the two response buttons was counterbalanced between (not within) Ss.

\section{RESULTS}

Two Ss in each of the four groups were excluded from data analysis as their performance accuracy deviated significantly from the desired level ( $5 \%$ and $15 \%$ for the accuracy and speed conditions, respectively). Therefore, the data reported represent an $\mathrm{N}=10$ for each group.

For each $S$, a measure of information transmitted $\left(\mathrm{H}_{\mathrm{t}}\right)$ was calculated across the six blocks of data collection. The $\mathrm{H}_{t}$ data then were subjected to an analysis of variance. The main effect of visual noise level was significant at $\mathrm{p}<.05, \mathrm{~F}(1,36)=$ 5.36 , and the main effect of accuracy level was significant at $p<.001$, $F(1,36)=565.97$. The Noise by Accuracy interaction was not significant, $F<1.0$. The average $\mathrm{H}_{t}$ under the noise-free condition was $.618 \mathrm{bit}$, while that with visual noise was .585 bit. Under the accuracy (5\% errors) condition, $\mathrm{H}_{\mathrm{t}}=. .771 \mathrm{bit}$, while under the speed (15\% errors) condition, it was .431 bit.

The reaction time data also were subjected to an analysis of variance.
Only correct response reaction times were included in the analysis, and correct "match" and correct "no-match" reaction times were pooled to enhance the reliability of the individual data points. This pooling is justified by a preliminary analysis which indicated no interaction between response type and either of the other two independent variables. As expected, "no-match" reaction times were slower than "match" reaction times (mean of medians, 569 and $512 \mathrm{msec}$, respectively).

Both main effects were significant at $\mathrm{p}<.001$ : for visual noise level, $F(1,36)=94.21$, and for accuracy level, $F(1,36)=18.72$. Further, the interaction of Noise by Accuracy was significant at $\mathrm{p}<.05: \mathrm{F}(1,36)=5.41$.

$$
\text { DISCUSSION }
$$

The significant interaction of Noise by Accuracy is direct evidence that the speed/accuracy tradeoff occurs in the initial stimulus encoding stage, and thus the earlier suggestion by Swanson \& Briggs (1969) on the locus of this effect is supported by the present data. Figure 1 illustrates the interaction where it may be noted that information transmitted $\left(H_{t}\right)$ serves as the measure of the accuracy levels actually obtained in this study.

Shown in Fig. 1 are least-squares fits of the equation

$$
R T=c+d\left(H_{t}\right)
$$

Use of a linear statement of additivity here is justified by the data of Swanson '\& Briggs (1969). The term d $\left(\mathrm{H}_{\mathrm{t}}\right)$ is an index of the speed/accuracy tradeoff, and as in the earlier research by Briggs and his students, this may be interpreted as the speed of sampling stimulus information from a short-term sensory store. The reciprocals of $d\left(H_{t}\right)$ provide estimates of this speed: $3.58 \mathrm{bits} / \mathrm{sec}$ and $10.87 \mathrm{bits} / \mathrm{sec}$ for the noise and the noise-free display conditions, respectively. The relative magnitudes of these two rate estimates are consistent with logic. One would expect a slower stimulus sampling process when dealing with a signal-in-noise display than when the display is essentially noise-free.

The noise-free rate of $10.87 \mathrm{bits} / \mathrm{sec}$ is somewhat faster than the rate estimated for stimulus sampling from previous data in this laboratory, e.g., rates of $6.4,6.7$, and $6.5 \mathrm{bits} / \mathrm{sec}$ have been found by Swanson \& Briggs (1969), Briggs \& Swanson (1970), and Lyons \& Briggs (1971), respectively, under noise-free display conditions. The faster rate noted here may be due in part to the fact that the present $S$ s worked with only a single size of positive set ( $M=4$ items in memory), whereas, in the previous research, sets of Size $M=1,2$, and 4 were experienced equally often by each $S$ across data collection. Thus, the present Ss were given substantially more experience with the $M=4$ condition, and apparently stimulus sampling rate can improve with practice on specific sizes of the positive set. Why this should be the case is not intuitively clear.

Now, it may be noted that Eq. 2 does not include a term for central processing time (see Eq. 1). Again, this is because only the $M=4$ condition was utilized here, and at least two different sizes of positive set are required to estimate the term $b$. An estimate of $b$ is available from another recent study performed at this laboratory with the same stimuli, positive sets, and test stimulus sequences used in the present study for the $M=4$ condition. In addition, $\mathrm{M}=1$ and $\mathrm{M}=2$ memory load conditions were utilized. Those data indicate that $b=7 \mathrm{msec} / \mathrm{bit}$ of uncertainty resolved at the central processing stage. Since $\mathrm{H}_{\mathrm{c}}=3.0 \mathrm{bits}$ for the $M=4$ condition of the present study, the intercept constants of Fig. 1 may be reduced by $210 \mathrm{msec}$ to provide the following additivity statements: for the noise-free condition, $\mathrm{RT}=.204+.092\left(\mathrm{H}_{\mathrm{t}}\right)+$ $.070\left(\mathrm{H}_{\mathrm{c}}\right)$, and for the visual noise condition, $\mathrm{RT}=.242+.279\left(\mathrm{H}_{\mathrm{t}}\right)+$ $.070\left(\mathrm{H}_{\mathrm{c}}\right)$. Utilizing the same value of $\mathrm{b}$ for both display conditions is justified by the results of both Stemberg (1967) and Bracey (1969).

To conclude, the following is offered by way of interpretation: (1) A single-letter stimulus appeared on the visual display. (2) More information than would be utilized 
was loaded into a short-term visual store and then sampled at a rate of $10.87 \mathrm{bits} / \mathrm{sec}$ under the noise-free condition but at a slower rate of $3.58 \mathrm{bits} / \mathrm{sec}$ under the visual noise condition. (3) The sampled information was then loaded into a buffer memory wherein some further filtering of signal from noise was undertaken, a process that required about $38 \mathrm{msec}$ (the difference between the c constants of Fig. 1). (4) From buffer memory (the final function of Stage 1), the information was transferred to Stage 2, where central testing of sampled stimulus features against memorial information on the positive and negative sets was carried out for purposes of stimulus classification. As indicated above, the estimated rate of $14.28 \mathrm{bits} / \mathrm{sec}$ for this central processing comes from $\mathrm{Ss}$ other than those used in the present study, but the other Ss were treated comparably to the present sample. (5) Following stimulus classification, response decoding occurred (Stage 3 ), and, finally, the selected response was executed.

\section{REFERENCES}

BRACEY, G. W. Two operations in character recognition: A partial replication. Perception \& Psychophysics. $1969,6,357-360$

BRIGGS, G. E.. \& SWANSON, J. M Encoding, decoding, and central functions in human information processing. Journal of Experimental Psychology, 1970, 86, 296-308.

LYONS, J. J. \& BRIGGS, G, E Speed-accuracy trade-off with different types of stimuli. Journal of Experimental
Psychology, 1971, 91, 115-119.

SMITH, E E Choice reaction time: An analysis of the major theoretical positions. Psychological Bulletin, 1968. 69. 77-110.

STERNBERG, S. High-speed scanning in human memory. Science, 1966, 153, 652-654.

STERNBERG, S. Two operations in character recognition: Some evidence from reaction-time measurements. Perception \& Psychophysics, 1967, 2, 45-53.

STERNBERG, S. The discovery of processing stages: Extensions of Donders method. Acta Psychologica, 1969, 30 , 276-315.

STERNBERG, S. Decomposing mental processes with reaction-time data. Invited address, Annual Meeting of the Midwestern Psychological Association, Detroit, May 1971.

SWANSON, J. M., \& BRIGGS, G. E. Information processing as a function of speed versus accuracy. Journal of Experimental Psychology, 1969, 81, 223-229. 